The Astrophysical Journal, 670:959-967, 2007 December 1

(C) 2007. The American Astronomical Society. All rights reserved. Printed in U.S.A.

\title{
NUCLEAR SPIRALS AS FEEDING CHANNELS TO THE SUPERMASSIVE BLACK HOLE: THE CASE OF THE GALAXY NGC 6951
}

\author{
Thaisa Storchi-Bergmann, Oli L. Dors, Jr., and Rogemar A. Riffel \\ Instituto de Física, Universidade Federal do Rio Grande do Sul, Av. Bento Gonçalves 9500, 91501-970 Porto Alegre RS, Brazil \\ KaMBIZ FATHI \\ Instituto de Astrofísica de Canarias, Vía Láctea s/n, 38200 La Laguna, Tenerife, Spain
}

David J. Axon and Andrew Robinson

Department of Physics, Rochester Institute of Technology, 85 Lomb Memorial Drive, Rochester, NY 14623

Alessandro Marconi

INAF-Osservatorio Astrofisico di Arcetri, Largo Fermi 5, 50125 Firenze, Italy

AND

GÖRAN ÖSTLIN

Stockholm Observatory, AlbaNova University Center, 10691 Stockholm, Sweden

Received 2007 March 23; accepted 2007 July 25

\begin{abstract}
We report the discovery of gas streaming motions along nuclear spiral arms toward the LINER nucleus of the galaxy NGC 6951. The observations, obtained using the GMOS integral field spectrograph on the Gemini North telescope, yielded maps of the flux distributions and gas kinematics in the $\mathrm{H} \alpha,[\mathrm{N} \mathrm{II}] \lambda 6584$, and [S II] $\lambda \lambda 6717,6731$ emission lines of the inner $7^{\prime \prime} \times 15^{\prime \prime}$ of the galaxy. This region includes a circumnuclear star-forming ring with a radius of $\sim 500 \mathrm{pc}$, a nuclear spiral inside the ring, and the LINER nucleus. The kinematics of the ionized gas is dominated by rotation, but subtraction of a kinematic model of a rotating exponential disk reveals deviations from circular rotation within the nuclear ring that can be attributed to (1) streaming motions along the nuclear spiral arms and (2) a bipolar outflow that seems to be associated with a nuclear jet. On the basis of the observed streaming velocities and geometry of the spiral arms, we estimate a mass inflow rate of ionized gas of $\approx 3 \times 10^{-4} M_{\odot} \mathrm{yr}^{-1}$, which is on the order of the accretion rate necessary to power the LINER nucleus of NGC 6951. Similar streaming motions toward the nucleus of another galaxy with a LINER nucleus, NGC 1097, have been reported by our group in a previous paper. Taken together, these results support a scenario in which nuclear spirals are channels through which matter is transferred from galactic scales to the nuclear region to feed the supermassive black hole.
\end{abstract}

Subject headings: galaxies: active — galaxies: individual (NGC 6951) — galaxies: kinematics and dynamics — galaxies: nuclei

\section{INTRODUCTION}

One long-standing problem in the study of nuclear activity in galaxies is to understand how mass is transferred from galactic scales down to nuclear scales to feed the supermassive black hole (SMBH) inside. Many theoretical studies and simulations (Shlosman et al. 1990; Emsellem et al. 2003; Knapen 2005; Emsellem et al. 2006) have shown that nonaxisymmetric potentials efficiently promote gas inflow toward the inner regions (Englmaier \& Shlosman 2004). Recent observations have revealed that structures such as small-scale disks or nuclear bars and associated spiral arms are frequently observed in the inner kiloparsec of active galaxies (Erwin \& Sparke 1999; Pogge \& Martini 2002; Laine et al. 2003).

In a recent work, Simões Lopes et al. (2007) have shown a strong correlation between the presence of nuclear dust structures (filaments, spirals, and disks) and activity in galaxies. Nuclear spirals, in particular, are estimated to reside in more than half of active galaxies (Martini et al. 2003). Martini \& Pogge (1999) have shown that nuclear spirals are not self-gravitating and that they are likely to be shocks in nuclear gas disks. Simulations by Maciejewski (2004a, 2004b) demonstrated that, if a central SMBH is present, spiral shocks can extend all the way to the SMBH vicinity and generate gas inflow consistent with the accretion rates inferred in local AGNs.
The above studies support the hypothesis that nuclear spirals are a mechanism for fueling the nuclear SMBH, transporting gas from kiloparsec scales down to a few tens of parsecs from the active nucleus (Knapen et al. 2000; Emsellem et al. 2001; Maciejewski et al. 2002; Marconi et al. 2003; Crenshaw et al. 2003; Fathi et al. 2005). This hypothesis has recently been confirmed by our group (Fathi et al. 2006) in the specific case of the LINER/Seyfert 1 galaxy NGC 1097. Using integral field spectroscopy at the Gemini telescope, we mapped the velocity field of the ionized gas and detected streaming motions toward the nucleus along nuclear spiral arms.

NGC 1097 is the only case so far in which streaming motions along nuclear spiral arms have been mapped. In order to verify if such spirals are always associated with inward streaming motions, it is necessary to map the gas kinematics in more galaxies. With this goal in mind, we have obtained Gemini integral field spectroscopic observations of a few more active galaxies with nuclear spirals observed in Hubble Space Telescope (HST) images. The sample was selected from nearby galaxies $(z<0.005)$ with intermediate inclinations to facilitate study of the gas kinematics on linear distance scales of tens of parsecs.

In the present paper we report the results for NGC 6951, a galaxy with Hubble type SAB(rs)bc, at a distance of $24 \mathrm{Mpc}$ (Tully 1988), such that $1^{\prime \prime}$ corresponds to $96 \mathrm{pc}$ at the galaxy. Originally it was argued that NGC 6951 has a LINER-type nucleus (Filippenko 
\& Sargent 1985), but more recently it has been suggested that actually its activity is intermediate between LINER and Seyfert (Pérez et al. 2000). NGC 6951 hosts a large-scale bar (with a total extent of $\sim 4 \mathrm{kpc}$ ), and at about $5^{\prime \prime}(480 \mathrm{pc}$ ) from the nucleus, there is a conspicuous star-forming ring, previously observed in $\mathrm{H} \alpha$ (Márquez \& Moles 1993; Pérez et al. 2000) and showing also strong $\mathrm{CO}$ and $\mathrm{HCN}$ emission (Kohno et al. 2000; Garcia-Burillo et al. 2005; Krips et al. 2007). Continuum radio emission has also been observed from the nucleus and star-forming ring by Saikia et al. (1994), while a higher resolution radio image can be found in Ho \& Ulvestad (2001).

Our Integral Field Unit (IFU) measurements show that, similarly to NGC 1097, the gas kinematics inside the nuclear ring of NGC 6951, although dominated by circular rotation, shows deviations in the measured radial velocities that can be interpreted as at least partially due to streaming inward motions along nuclear spiral arms. Our results strengthen the case that nuclear spirals are indeed channels to feed the supermassive black hole in active galaxies.

The present paper is organized as follows. In $\S 2$ we describe the observations and reductions. In $\S 3$ we present the flux and kinematic measurements. In $\S 4$ we discuss the results, and in $\S 5$ we present our conclusions.

\section{OBSERVATIONS AND REDUCTIONS}

The observations were obtained with the Integral Field Unit of the Gemini Multi-Object Spectrograph (GMOS IFU; AllingtonSmith et al. 2002) at the Gemini North telescope on the nights of 2006 August 31 and 2006 September 1 (program GN-2006BQ-23). The observations consisted of three adjacent IFU fields (covering $5^{\prime \prime} \times 7^{\prime \prime}$ each), resulting in a total angular coverage of $7^{\prime \prime} \times 15^{\prime \prime}$ around the nucleus. Three exposures of $500 \mathrm{~s}$ were obtained for each of the three IFU fields, slightly shifted in order to correct for detector defects. Observations of the three IFU fields were obtained consecutively on the same night. The fluxes in adjacent IFU pixels from neighboring fields were found to be consistent within $10 \%$. Therefore, no shifts or scaling were applied when combining the three fields to obtain the final mosaic.

The longest extent of the three adjacent IFU fields was oriented along position angle (P.A.) $140^{\circ}$, selected to approximately coincide with the major axis of the galaxy (P.A. $=138^{\circ}$, according to Márquez \& Moles 1993), while the large-scale bar is oriented approximately east-west. Each IFU fiber has a diameter that corresponds to $0.2^{\prime \prime}$ in the sky, and the seeing during the observations ranged between $0.4^{\prime \prime}$ and $0.5^{\prime \prime}$, corresponding to a spatial resolution at the galaxy of $\approx 40 \mathrm{pc}$.

The selected wavelength range was 5600-7000 $\AA$, in order to cover the $\mathrm{H} \alpha+[\mathrm{N}$ II] $\lambda \lambda 6548,6584$ and [S II] $\lambda \lambda 6716,6731$ emission lines, observed with the grating GMOS R400-G5303 (set to a central wavelength of $\approx 6300 \AA$ ), with an instrumental FWHM of $2.9 \AA$, corresponding to a spectral resolution of $R \approx 2300$ $\left(\approx 130 \mathrm{~km} \mathrm{~s}^{-1}\right)$.

The data reduction was performed using the specific tasks developed for GMOS data in the gemini.gmos package, as well as generic tasks in IRAF. The reduction process comprised bias subtraction, flat-fielding, trimming, wavelength calibration, sky subtraction, relative flux calibration, building of the data cubes at a sampling of $0.1^{\prime \prime} \times 0.1^{\prime \prime}$, and finally the alignment and combination of the nine data cubes. As we have obtained only relative flux calibration (what is called in Gemini "baseline calibrations"), we have normalized the flux levels using the $\mathrm{H} \alpha$ nuclear flux reported by Pérez et al. (2000).

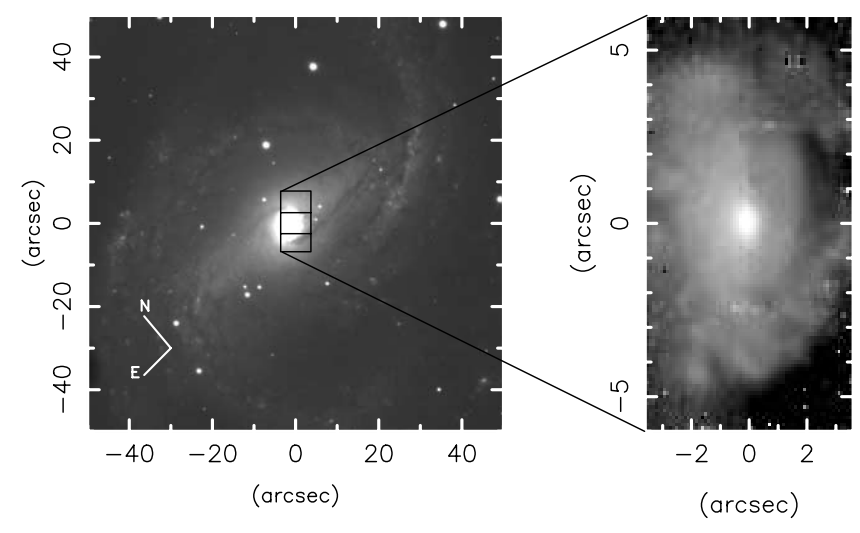

FIG. 1.-Left: Acquisition image in the $R$ band, showing the three IFU fields covered by the observations. Right: Image obtained from the IFU spectra in the continuum adjacent to the $\mathrm{H} \alpha+\left[\mathrm{N}_{\text {II }}\right]$ emission lines. The sampling of the data is $0.1^{\prime \prime} \times 0.1^{\prime \prime}$, and the seeing was $\approx 0.4^{\prime \prime}$.

\section{RESULTS}

In Figure 1 we show the acquisition image with the location of the three IFU fields, together with an image obtained from the IFU data in the continuum adjacent to the $\mathrm{H} \alpha$ emission line. The IFU observations cover the nuclear region out to the star-forming ring, which can be more clearly seen in the $\mathrm{H} \alpha$ and [ $\left.\mathrm{N}_{\text {II }}\right]$ images shown below in Figure 3. The $\mathrm{H} \alpha$ and [ $\mathrm{N}$ II] emission lines could be measured over most of the $7^{\prime \prime} \times 15^{\prime \prime}$ field, except for a few locations that appear in black in Figure 3. For the [S II] emission lines, there are more locations at which the lines could not be measured due to the low signal-to-noise ratio in these lines.

In Figure 2 we show a sample of spectra: one from the nucleus, one from the star-forming ring, and two from locations between the nucleus and the ring. The emission lines present in most spectra are $\mathrm{H} \alpha,[\mathrm{N}$ II $] \lambda \lambda 6548,6584$, and $[\mathrm{S}$ II $] \lambda \lambda 6717,6731$. The lines $\left[\mathrm{O}_{\mathrm{I}}\right] \lambda \lambda 6300,6363$ were marginally detected only in a few spectra and were not considered in our analysis due to the poor signal-tonoise ratio (in these lines). The emission-line spectrum from the nucleus is LINER-like ([N II] $\lambda 6584$ stronger than $\mathrm{H} \alpha$ ), as was already known from previous works (e.g., Filippenko \& Sargent 1985). More recently, Ho et al. (1997) have argued that the nuclear activity is better classified as Seyfert 2 after an analysis of the nuclear spectrum that took into account the effect of the underlying stellar absorption in the fluxes of the Balmer lines. Pérez et al. (2000), after a similar analysis, conclude that the nuclear activity of NGC 6951 is intermediate between LINER and Seyfert 2. Our data are consistent with all these classifications, as our restricted wavelength coverage does not allow the observation of additional line ratios necessary to distinguish between the different types of activity. The emission-line ratios in the star-forming ring are typical of $\mathrm{H}$ in regions, while in the regions between the nucleus and the ring, the line ratios are also mostly LINER-like, becoming "H II-like" as the star-forming ring is approached.

\subsection{Line Fluxes and Excitation of the Emitting Gas}

The line fluxes, radial velocities, and velocity dispersions were obtained by fitting Gaussians to the emission lines, which accurately represent the observed line profiles. The $\mathrm{H} \alpha$ image obtained from the integrated $\mathrm{H} \alpha$ flux is shown in Figure 3, together with the image in the adjacent continuum. Most spectra have signalto-noise ratios in the $\mathrm{H} \alpha$ and $[\mathrm{N}$ II] emission lines that are larger than 50 . 


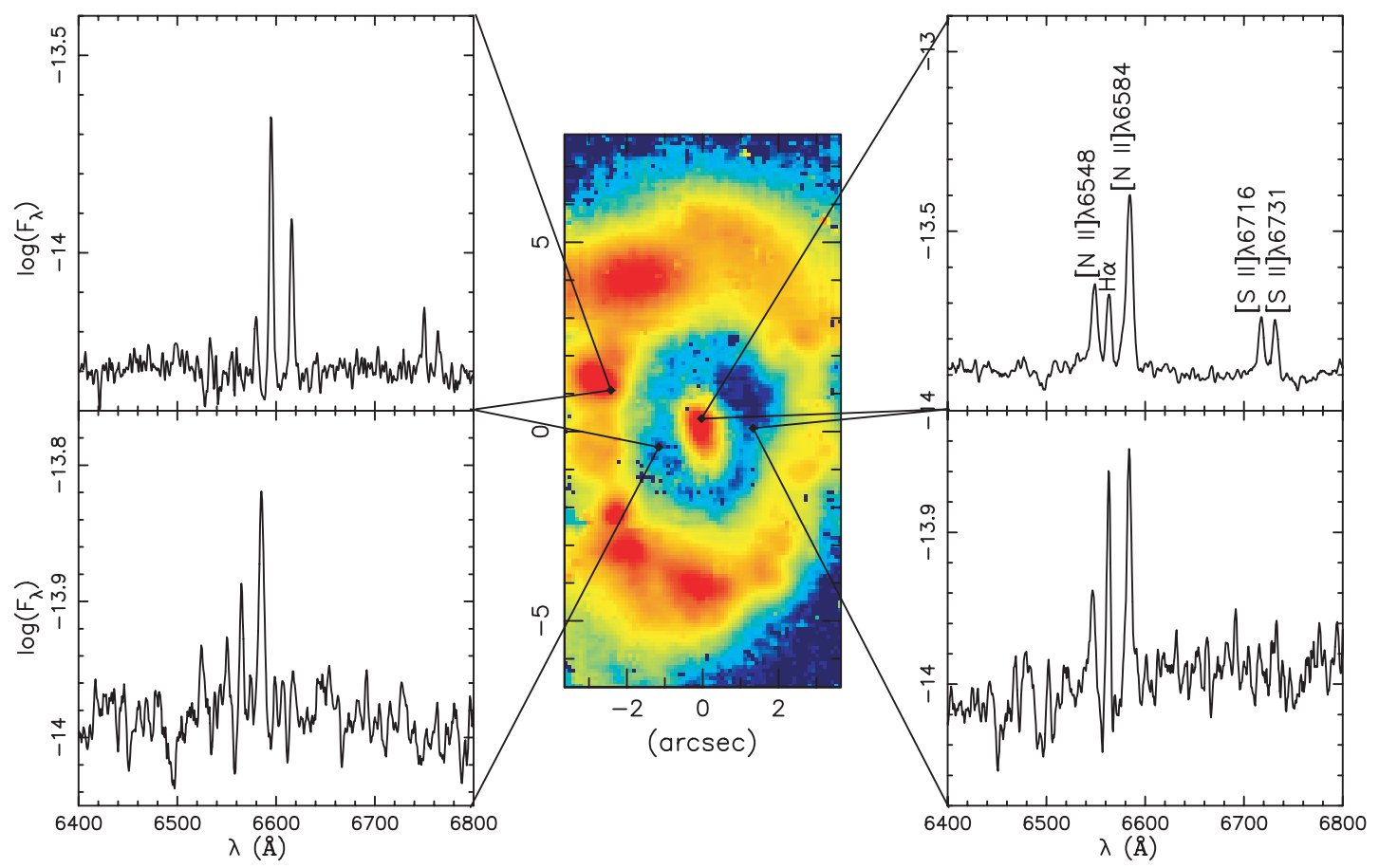

FIG. 2.- Sample of spectra, with the corresponding locations identified in the IFU image obtained from the integrated flux in the H $\alpha$ emission line (middle). Top right, The nuclear spectrum; top left, a spectrum from the star-forming ring; bottom left and bottom right, spectra from regions between the nucleus and the star-forming ring.

The star-forming ring is clearly seen in the $\mathrm{H} \alpha$ image, formed by several $\mathrm{H}$ II regions that appear as emission-line knots along the ring. The ring is more extended along the major axis of the galaxy (P.A. $\sim 140^{\circ}$ ), where it reaches $\approx 4.5^{\prime \prime}$ from the nucleus ( $430 \mathrm{pc}$ at the galaxy), while in the perpendicular direction it reaches only $\approx 2.5^{\prime \prime}$.

The line ratio map of $[\mathrm{N} \mathrm{II}] / \mathrm{H} \alpha$, obtained from the ratio of the images in [N $\mathrm{NI}] \lambda 6584$ and $\mathrm{H} \alpha$, as well as the [S II] $\lambda 6717 / \lambda 6731$ ratio map, is also shown in Figure 3. Prior to the construction of the $[\mathrm{N}$ II] $/ \mathrm{H} \alpha$ map, we have corrected the $\mathrm{H} \alpha$ line fluxes by an average underlying absorption due to the stellar population with an equivalent width of $0.75 \AA$. This procedure is necessary in order to avoid unphysical very high ratios of $[\mathrm{N} \mathrm{II}] / \mathrm{H} \alpha$ and is supported by the inspection of a few spectra, which reveal that where
$\mathrm{H} \alpha$ emission is very faint, the line is indeed obviously filling an absorption. The average value of $0.75 \AA$ was adopted from the work of Pérez et al. (2000), who estimated an absorption equivalent width of $\mathrm{H} \alpha$ of $1 \AA$ at the nucleus and $0.5 \AA$ at the ring on the basis of the analysis of the stellar population. We thus decided to adopt an average value between that of the nucleus and that of the ring, as the resulting corrected fluxes did not change by more than $\sim 20 \%$ at the nucleus and $\sim 10 \%$ at the ring if we change the underlying absorption by $0.25 \AA$. The highest $[\mathrm{N} \mathrm{II}] / \mathrm{H} \alpha$ line ratios reach values of $4-5$ within $\mathrm{a} \approx 1^{\prime \prime}$ radius from the nucleus, decreasing to $2-3$ between $1^{\prime \prime}$ and $2^{\prime \prime}$ and down to $\approx 0.3$ at the circumnuclear ring.

The ratio $\left[\mathrm{S}_{\mathrm{II}}\right] \lambda 6717 / \lambda 6731$ reaches a minimum value of $\approx 0.8$, implying a maximum gas density of $\approx 10^{3} \mathrm{~cm}^{-3}$ within $\approx 1^{\prime \prime}$ from
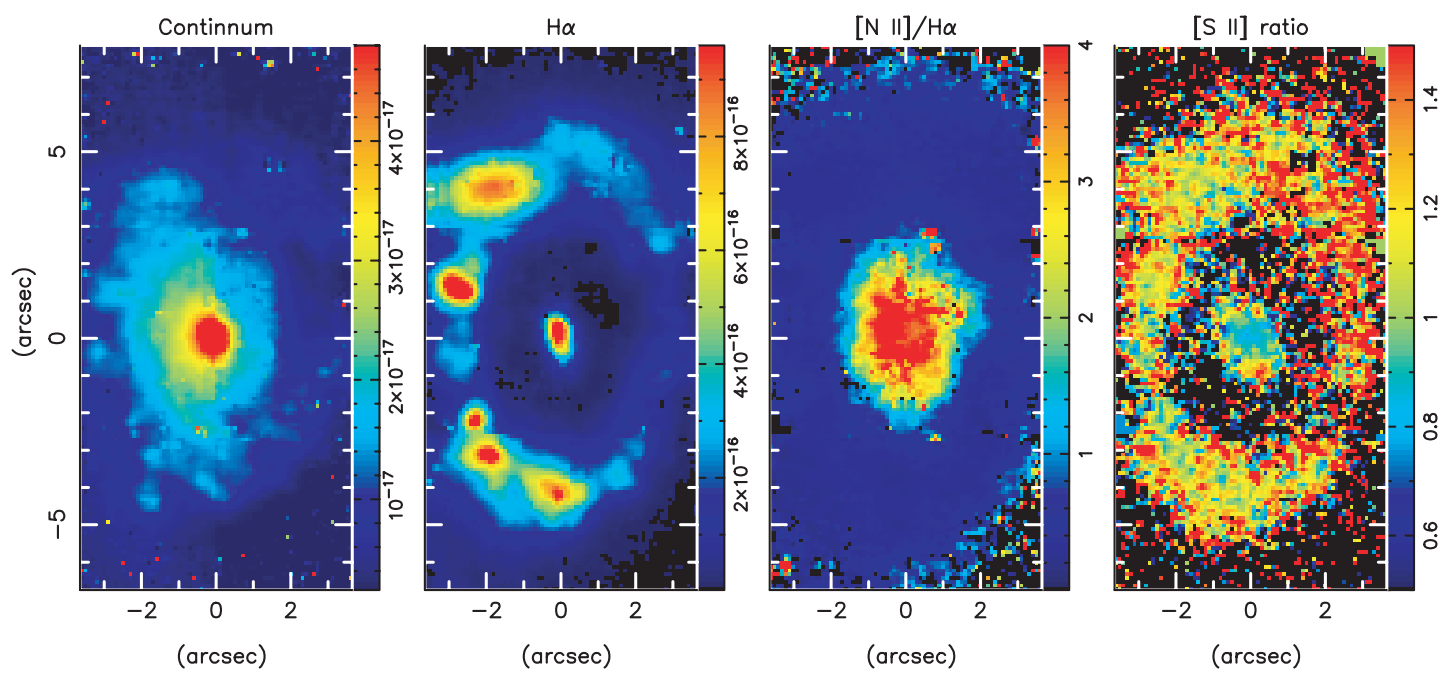

Fig. 3.-From left to right: Map of the continuum flux adjacent to $\mathrm{H} \alpha$ (in units of $\mathrm{erg} \mathrm{cm}^{-2} \mathrm{~s}^{-1} \AA^{-1}$ pixel $^{-1}$ ); map of the $\mathrm{H} \alpha$ integrated flux (in units of erg cm ${ }^{-2} \mathrm{~s}^{-1}$ pixel $^{-1}$ ); and line ratio maps for $[\mathrm{N} \mathrm{II]} / \mathrm{H} \alpha$ and [S II] $\lambda 6717 / \lambda 6731$. 
the nucleus (Osterbrock 1989). At the star-forming ring, the [S II] ratio ranges from 1 to 1.4 , with typical values at most locations of between 1.1 and 1.2, implying gas densities in the range 250$400 \mathrm{~cm}^{-3}$. At the borders of the ring, the low-density limit value of $1.4\left(<100 \mathrm{~cm}^{-3}\right)$ is reached. In most locations between the nucleus and the ring, the signal-to-noise ratio was too low to get a reliable value for the $[\mathrm{S}$ II] ratio. In many locations where it could be measured, it was in the low-density limit. In order to investigate this further, we have binned nine spectra together (which corresponds to $0.3^{\prime \prime} \times 0.3^{\prime \prime}$, and thus approximately to the angular resolution of the data) in several locations and thereby got ratios in the range $1.2<\left[\mathrm{S}_{\mathrm{II}}\right] \lambda 6717 / \lambda 6731<1.4$, somewhat above the low-density limit, implying ionized gas densities of $\sim 100 \mathrm{~cm}^{-3}$, which we will adopt as the typical value for this region.

\subsection{Kinematics}

The central wavelengths of the Gaussian curves fitted to the emission lines were used to obtain the gas radial velocities, while the full widths at half-maximum (FWHMs) were used to obtain the gas velocity dispersions ( $\sigma=\mathrm{FWHM} / 2.355)$. The accuracy of the velocity measurements is $\approx 10 \mathrm{~km} \mathrm{~s}^{-1}$. The radial velocity field obtained from $\mathrm{H} \alpha$ is shown in the top left panel of Figure 4. It is dominated by rotation, as evidenced by its similarity to the classical "spider diagram" (Binney \& Merrifield 1998). (The pixels for which we could not measure the kinematics due to low signalto-noise ratio in the emission lines are shown in black in the figure.)

There are, nevertheless, clear deviations from simple rotation in the radial velocity field. In order to isolate these deviations, we have fitted an exponential thin-disk kinematic model to the $\mathrm{H} \alpha$ radial velocity data, assuming that the density profile is exponential and that its kinematics is circular rotation (Freeman 1970; Binney \& Tremaine 1987). The procedure is similar to the one we have adopted in modeling the circumnuclear gas kinematics of NGC 1097 (Fathi et al. 2005, 2006).

The velocity field corresponding to the best fit is shown in the top right panel of Figure 4. The fit to the model gives a systemic velocity of $1450 \pm 20 \mathrm{~km} \mathrm{~s}^{-1}$, a deprojected maximum velocity amplitude of $220 \pm 10 \mathrm{~km} \mathrm{~s}^{-1}$, a disk scale length of $4.1^{\prime \prime} \pm$ $0.3^{\prime \prime}$ (390 pc at the galaxy), and a position angle for the line of nodes of P.A. $=125^{\circ} \pm 10^{\circ}$. We have adopted a disk inclination of $i=42^{\circ}$, a value derived from photometry (Márquez \& Moles 1993), because the inclination is not well constrained in our multiparameter space-fitting procedure.

A comparison between the measured radial velocity field and the disk model reveals deviations from simple rotation. These deviations are observed along the star-forming ring, as well as inside the ring, where, in particular, we have identified two features resembling partial spiral arms that are marked by two white curved lines with arrows in Figure 4. In order to investigate if these deviations correspond to real features in the galaxy, we have constructed a structure map (Pogge \& Martini 2002) using an HST image of the nuclear region of NGC 6951 obtained through the filter F606W (HST proposal 8597; PI M. Regan). The structure map is useful to enhance the contrast in the images, thus showing more clearly both dark structures - regions obscured by dustand bright regions such as $\mathrm{H}$ II regions, a compact bright nucleus, or star clusters. The structure map is shown in the bottom left panel of Figure 4, where we have drawn the same white lines as in the top left panel, showing that the partial spiral arms follow the dusty spiral structure seen in the structure map. Although there are more spiral arms in the structure map than the two we see in the velocity residual map, this difference can be understood as due to the poorer angular resolution of the IFU data $\left(\approx 0.4^{\prime \prime}\right)$ compared to that of the HST $\left(0.05^{\prime \prime}\right)$, which precludes us from establishing
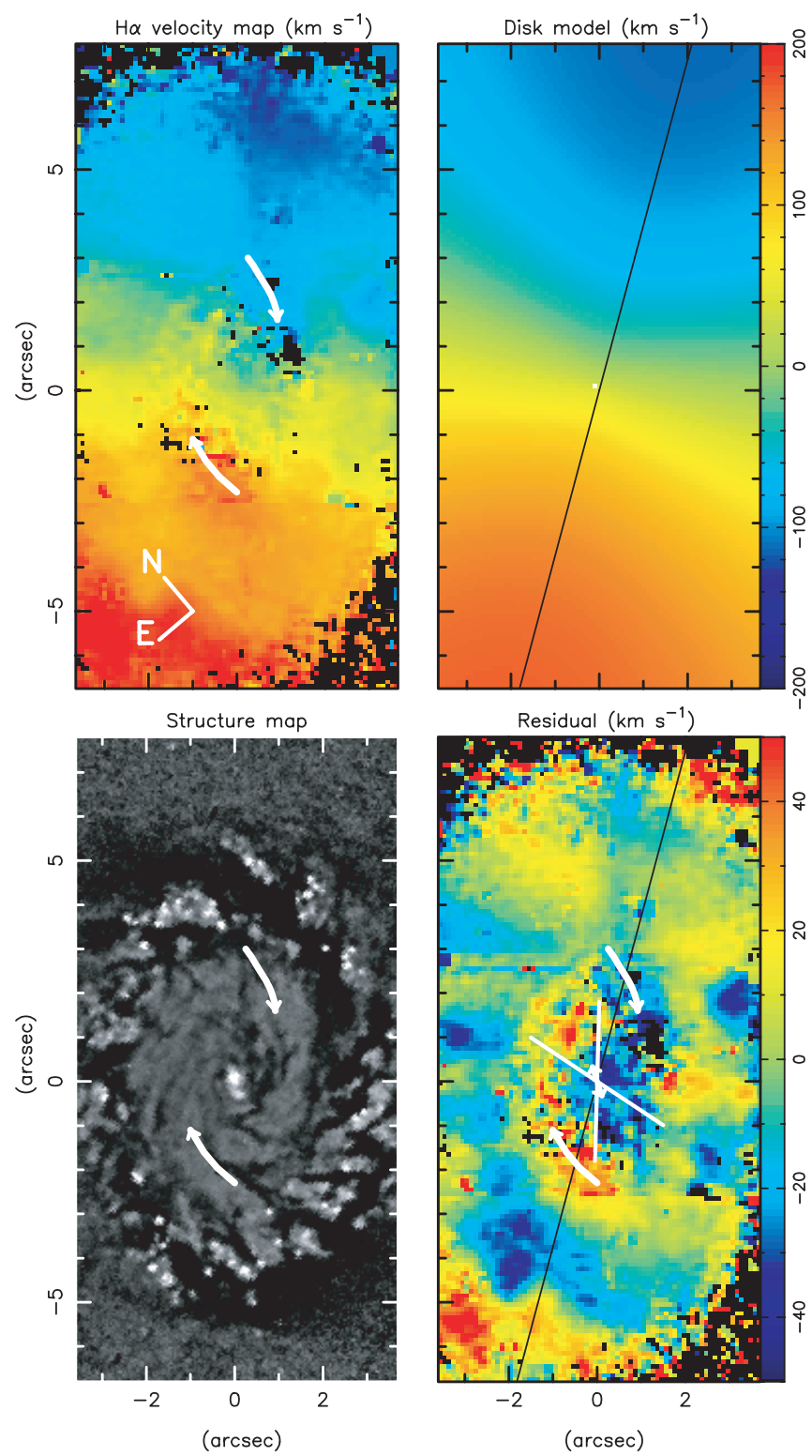

FIG. 4.-Top left: $\mathrm{H} \alpha$ velocity field. Top right: Model velocity field. Bottom left: Structure map. Bottom right: Difference between observed and model velocity fields. Curved lines with arrows identify two velocity structures that deviate from the model: the "west arm" (upper) and the "east arm" (lower). The small bar with arrows at the nucleus in the bottom right panel represents a compact radio source (Saikia et al. 2002), and the two white lines delineate a bicone within which we observe an enhancement in the values of $\sigma$ (see Fig. 5). The straight black line shows the location of the line of nodes.

a more detailed correspondence. In the bottom right panel of Figure 4 we show the residuals between the observed and the model velocity field, where the white lines correspond again to the partial arm structures. The upper arm (hereafter called the west arm) corresponds to a blueshifted region in the residual map, while the lower arm (hereafter the east arm) corresponds to a redshifted region. There are additional blue- and redshift residuals in the star-forming ring, as well as close to the nucleus. The additional velocity residuals close to the nucleus in Figure 4 are approximately delimited by the borders of a "bicone," sketched in white in the figure, which corresponds to regions in which we see an increase in the values of the velocity dispersion, as discussed below.

The gas velocity dispersion $(\sigma)$ maps in $\mathrm{H} \alpha$ and [N $\mathrm{NI}$ ] are shown in Figure 5. The instrumental value of $\sigma$ is $\approx 50 \mathrm{~km} \mathrm{~s}^{-1}$; thus, it can 

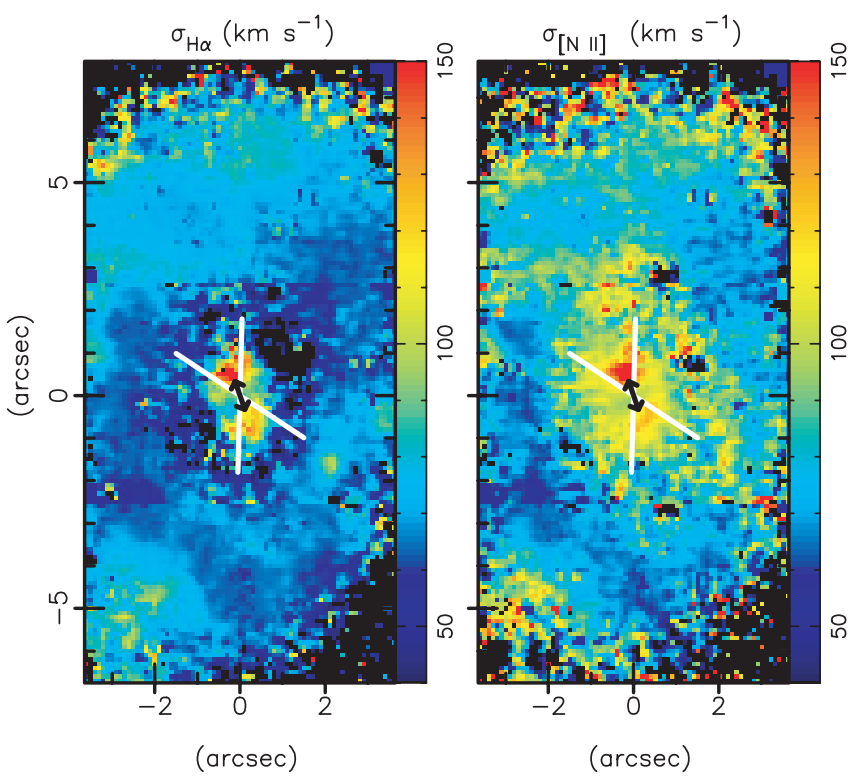

FIG. 5.- Velocity dispersion maps in $\mathrm{H} \alpha($ left $)$ and [ $\left.\mathrm{N}_{\mathrm{II}}\right] \lambda 6584$ (right). The black bar with two arrows represents the compact nuclear radio source (Saikia et al. 2002), while the two white lines delineate the borders of a bicone within which the highest $\sigma$-values are observed, probably due to stirring of the circumnuclear ISM by a nuclear radio jet.

be concluded that many values, particularly for $\mathrm{H} \alpha$, are at the resolution limit of the instrument. The low values obtained for $\mathrm{H} \alpha$ in some regions are due to the fact that in the region between the nucleus and the star-forming ring, $\mathrm{H} \alpha$ emission is strongly affected by the underlying stellar absorption (discussed above), which makes the observed emission line weaker and narrower. On the other hand, the [ $\mathrm{N}$ II] profile provides a reliable measure of $\sigma$ because it is free of the contamination by stellar absorption. Most $\sigma$-values range between 60 and $80 \mathrm{~km} \mathrm{~s}^{-1}$, but there are larger values reaching $140 \mathrm{~km} \mathrm{~s}^{-1}$ in two blobs within $\approx 1^{\prime \prime}$ of the nucleus to the south-southeast and north-northwest.

What could be the origin of these enhancements in $\sigma$ ? Our experience with previous IFU observations (of other targets; e.g., Riffel et al. 2006; F. Barbosa et al. 2008, in preparation) reveals that increases in $\sigma$ in the gas surrounding AGNs are usually related to the presence of a passing radio jet. We searched the literature for a possible nuclear radio jet and found that VLA observations by Saikia et al. (2002) of the inner region of NGC 6951 indeed suggest the presence of a resolved nuclear radio source presenting an angular size of $0.7^{\prime \prime} \times 0.2^{\prime \prime}$ along P.A. $=156^{\circ}$. We show a representation of this small radio source as a bar with arrows in Figure 5, as well as in the bottom right panel of Figure 4. Note that the extent and orientation of this radio structure coincide with those of a bright similarly elongated nuclear structure seen in the structure map (Fig. 4, bottom left). As the F606W filter used to obtain the HST image includes $\mathrm{H} \alpha$, this coincidence suggests that we are resolving enhanced $\mathrm{H} \alpha$ emission associated with the radio structure in this image.

It can be seen that the estimated extent of the radio source fits well in between the two regions of enhanced $\sigma$-values in Figure 5 . We have sketched (in white in the figure) the borders of a bicone that includes the regions of the largest $\sigma$-values. We note that the corresponding locations in Figure 4 approximately delimit also a redshifted $\mathrm{H} \alpha$-emitting region to the north-northwest and a blueshifted region to the south-southeast observed in the residual $\mathrm{H} \alpha$ velocity map (Fig. 4, bottom right). The observed increase in $\sigma$ along the bicone and the presence of associated blue- and redshifts suggests that these two structures are due to an outflow driven by the radio jet. The delineated bicone in Figures 4 and 5 approximately separates the regions affected by the outflow from the regions affected by the residual motion along the spiral arms.

\section{DISCUSSION}

\subsection{Excitation}

An extensive photometric and spectroscopic study of the galaxy NGC 6951 has been presented by Pérez et al. (2000, hereafter P00). They adopt the disk major axis to be P.A. $=138^{\circ}$ and have obtained long-slit spectra (slit width of $1^{\prime \prime}$ ) along this P.A., as well as along P.A. $=48^{\circ}$ (minor axis) and P.A. $=84^{\circ}$.

P00 quote line ratio values of $[\mathrm{N}$ II $] / \mathrm{H} \alpha$ larger than unity within the inner $2^{\prime \prime}$, reaching $\approx 5$ at the nucleus, in agreement with our results. They present data within the nuclear region at a sampling of $0.5^{\prime \prime}$ (although the seeing was $1.2^{\prime \prime}$ during their observations). P00 report electron density values increasing from $N_{e} \sim 300 \mathrm{~cm}^{-3}$ at the ring to $\sim 1000 \mathrm{~cm}^{-3}$ at the nucleus, also in agreement with our results.

In order to further investigate the excitation, we have plotted in Figure 6 the ratio $[\mathrm{N} \mathrm{II]} / \mathrm{H} \alpha$ versus the velocity dispersion obtained from the width of the [N $\left.\mathrm{N}_{\mathrm{II}}\right] \lambda 6584$ emission line, $\sigma_{\left[\mathrm{N}_{\mathrm{II}}\right]}$. We have separated the data from the ring and from the region inside the ring, using the $\mathrm{H} \alpha$ image to identify the region dominated by the ring. In order to do this, we have fitted two ellipses, one to the inner border of the ring and another to the outer border. Along the longest extent of the ring (approximately along the vertical axis of the figures), the inner border is located at $2.8^{\prime \prime}$ from the nucleus and the outer border at $6.8^{\prime \prime}$, while along the shortest extent of the ring the inner border is at $2.0^{\prime \prime}$ and the outer border at $3.6^{\prime \prime}$.

It can be seen that the behavior of the two regions is clearly different: while for the ring itself, most values of $\left[\mathrm{N}_{\mathrm{II}}\right] / \mathrm{H} \alpha$ are in the range $0.3-0.4$, typical of $\mathrm{H}$ II regions, and show just a weak correlation with $\sigma_{[\mathrm{NI}}$, in the region inside the ring, the [N II] $/ \mathrm{H} \alpha$ values are much higher and present a strong correlation with $\sigma_{[\mathrm{NI}]}$. This behavior has been observed previously in other galaxies (e.g., Keppel et al. 1991; Sokolowski et al. 1991; Storchi-Bergmann et al. 1996), indicating that the gas excitation is related to its kinematics, and it suggests in particular that at least part of the ionization is produced by shocks, as shocks do enhance the $[\mathrm{N} \mathrm{II]} / \mathrm{H} \alpha$ ratio (Viegas \& Contini 1994; Sutherland et al. 1993), as well as broaden the emission lines. The presence of shocks in the vicinity of the nucleus is particularly evident in Figure 5, where we have shown that the two regions that have the highest observed $\sigma$-values are located at the ends of the nuclear radio structure and can be interpreted as gas from the interstellar medium (ISM) of the galaxy shocked by a radio jet.

\subsection{The Nuclear Spiral}

Garcia-Burillo et al. (2005, hereafter GB05) have obtained molecular CO radio images of the inner region of NGC 6951, showing that most of the $\mathrm{CO}$ emission is concentrated in two winding nuclear spiral arms that end up as a pseudoring at $\sim 350 \mathrm{pc}$ from the nucleus, with an estimated molecular gas mass of $\approx 3 \times 10^{8} \mathrm{M} \odot$. This nuclear spiral seen in molecular gas corresponds in fact to the $\mathrm{H} \alpha$ ring, whose strongest $\mathrm{H} \alpha$ emission seems to be a bit external to the strongest molecular gas emission.

GB05 also report the observation of "an unresolved molecular complex" within $50 \mathrm{pc}$ from the nucleus, estimating its mass to be a few times $\sim 10^{6} M_{\odot}$. The presence of a relatively large molecular gas mass at the nucleus is supported also by the recent millimeter interferometric observations by Krips et al. (2007), 


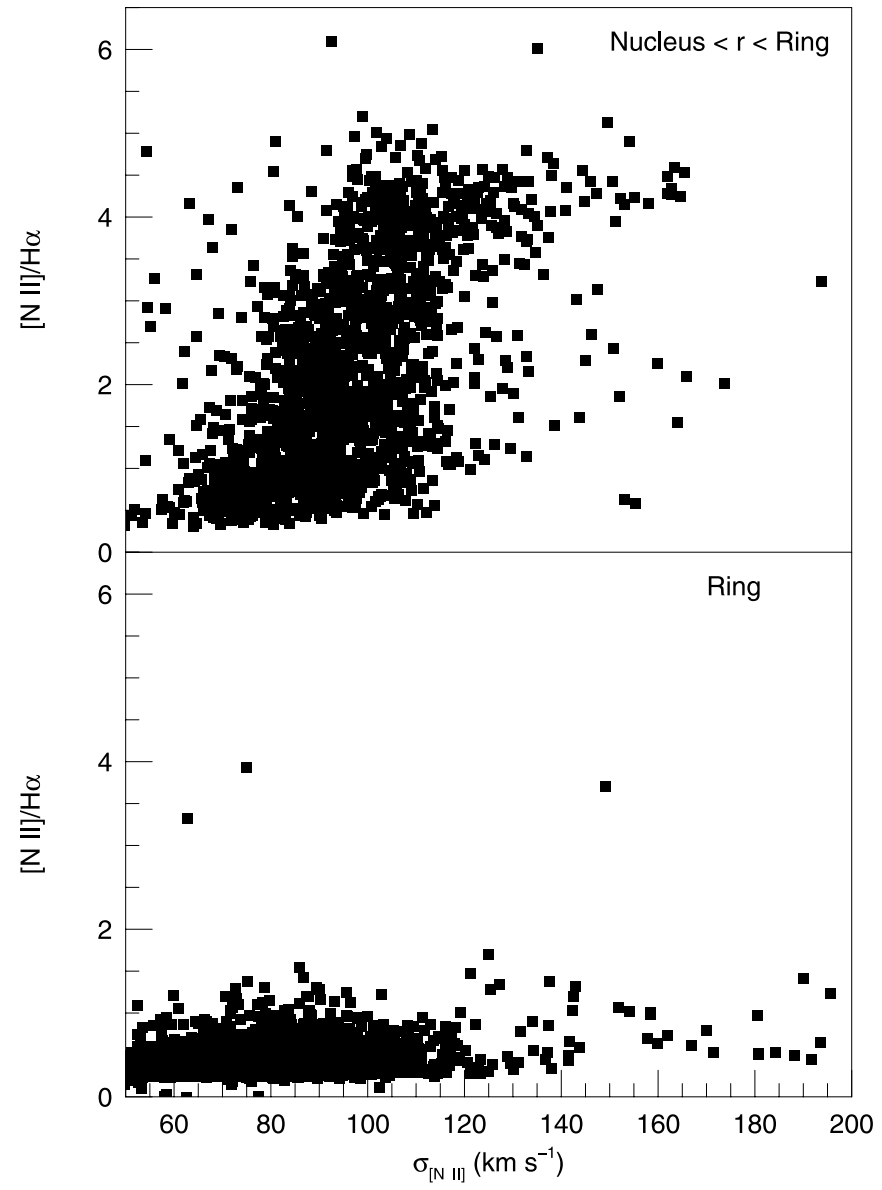

FIG. 6.-Ratio of [ $\left.\mathrm{N}_{\mathrm{II}}\right] \lambda 6584 / \mathrm{H} \alpha$ as a function of the [ $\left.\mathrm{N}_{\mathrm{II}}\right] \lambda 6584$ velocity dispersion for the region inside the ring (top) and for the ring itself (bottom).

who have found compact $\mathrm{HCN}$ emission at the nucleus and estimate an even larger mass for the corresponding molecular complex of $\sim(2-10) \times 10^{7} M_{\odot}$. These observations support the recent inflow of molecular gas to the nuclear region.

Besides the nuclear complex, GB05 have also "tentatively detected" (their words) low-level CO emission bridging the gap between the nuclear source and the north part of the ring, with an estimated molecular mass of $\sim 10^{7} M_{\odot}$. GB05 argue that this structure could be the molecular counterpart of the filamentary spiral structure identified in a $J-H$ color map of NGC 6951 obtained from $H S T$ images. Their $J-H$ map (Fig. $4 a$ in GB05) is very similar to the structure map that we show in the bottom left panel of Figure 4. A comparison of this structure map with both our $\mathrm{H} \alpha$ velocity map (Fig. 4, top left) and Figure 4 of GB05 shows that their "molecular bridge" seems to partially coincide with the west arm structure seen in our kinematic map, particularly with the "inner end" of our partial west arm. A close look shows that the molecular bridge seems to be associated with the darker regions of the structure map closer to the nucleus (and thus with the dust structure), while our west partial arm is more closely coincident with the brighter portions of the structure map, a bit more distant from the nucleus. A possible interpretation is that we are seeing two parts of a spiral arm: a more external one, traced by $\mathrm{H} \alpha$ emission, and a more internal one, seen only in molecular gas emission, probably due to the absence or faintness of ionized gas emission.

\subsection{Kinematics}

The rotation pattern in the $\mathrm{H} \alpha$ radial velocity field (Fig. 4) shows that the southeast side of the galaxy is receding, while the northwest side is approaching. Under the assumption that the spiral arms are trailing and that the approaching side of the bar piles up the dust in front of it, it can be concluded that the near side of the galaxy is the southwest side and the far side is the northeast side. The deviations from circular rotation along the two partial spiral arms in the inner $2^{\prime \prime}-3^{\prime \prime}$ from the nucleus - the blueshifted residuals along the west arm and the redshifted residuals along the east arm — can thus be interpreted as streaming motions along nuclear spiral arms toward the nucleus.

Nevertheless, the residuals in this inner region extend beyond the region covered by the east and west arms drawn in Figure 4: in particular, there are additional blueshifts observed to the southsoutheast of the nucleus and redshifts observed to the northnorthwest, which seem to be a continuation of the east and west arms. Nevertheless, at $\sim 1^{\prime \prime}$ north-northwest and south-southeast of the nucleus, they are not consistent with streaming motions toward the nucleus. We conclude that these residuals are in fact outflows due to the interaction of the radio source at the nucleus (Saikia et al. 2002) with the circumnuclear ISM of the galaxy.

This hypothesis is supported by the increase in the velocity dispersion of the gas from 70 to $140 \mathrm{~km} \mathrm{~s}^{-1}$ along the position angle of the radio structure (P.A. $=156^{\circ}$ ), approximately within a region delineated by two white lines representing the borders of a bicone shown in Figure 5. From the location of this bicone in Figure 4, we conclude that the residual blueshifts at $\approx 1^{\prime \prime}$ north-northwest and redshifts at $\approx 1^{\prime \prime}$ south-southeast of the nucleus are observed in the regions corresponding to those with enhanced values of $\sigma$ and can be interpreted as outflows related to the radio source.

The residuals from circular motion in the inner $3^{\prime \prime}$ thus include two components: (1) streaming motions toward the nucleus along spiral arms and (2) an outflow due to the interaction of a radio source with the circumnuclear ISM of the galaxy. From the relative intensity of the continuum observed in the structure map at the location of the radio structure, which is brighter to the side to which the residuals are blueshifts, we suggest that the near side of the jet is projected against the near side of the galaxy, which is possible only if its angle relative to the galaxy plane is smaller than $48^{\circ}$ for the adopted inclination of the galaxy of $42^{\circ}$. In the regions corresponding to the ring, there are also radial velocity residuals relative to the circular rotation that could be due to outflows from the starbursts along the ring and/or deviations due to the accumulation of the gas in the inner Lindblad resonance (ILR).

The kinematics we have obtained for the nuclear region of NGC 6951 can be compared with that obtained in previous works, in particular by P00, who have studied the large-scale kinematics from long-slit spectroscopy along three position angles (over a radius of up to $100^{\prime \prime}$ from the nucleus): one along the photometric major axis $\left(138^{\circ}\right)$, one along the perpendicular direction $\left(48^{\circ}\right)$, and one along P.A. $=84^{\circ}$. On the basis of the kinematics along these three position angles, P00 obtain a systemic velocity of $V_{\text {sys }}=1417 \pm 4 \mathrm{~km} \mathrm{~s}^{-1}$ and an amplitude of the rotation curve of $\approx 200 \mathrm{~km} \mathrm{~s}^{-1}$ after deprojection (see, for example, Fig. 13 of P00). These parameters are similar to our values of $V_{\text {sys }}=$ $1450 \pm 20 \mathrm{~km} \mathrm{~s}^{-1}$ and amplitude of $220 \pm 10 \mathrm{~km} \mathrm{~s}^{-1}$ of the rotation curve. These parameters are also similar to those previously obtained by Márquez \& Moles (1993) on the basis of long-slit spectra along the major and minor axes of the galaxy, in spite of the fact that our kinematic data are two-dimensional (2D) but cover a much smaller radial extent $\left(3.5^{\prime \prime} \times 7^{\prime \prime}\right)$. This difference in angular coverage may explain the difference between the majoraxis orientation obtained in previous studies and the one derived from our kinematic modeling of $125^{\circ} \pm 10^{\circ}$.

P00 report a "peculiar structure" in the velocity field along the minor axis $\left(\right.$ P.A. $=48^{\circ}$ ), which they argue could be due to a 
nuclear outflow or to a structure related to the "inner ILR (iILR)." They adopt the second hypothesis, suggesting counterrotation of an inner disk, which they argue could be related to the iILR, which is calculated to occur at $180 \mathrm{pc}\left(2^{\prime \prime}\right)$. They argue that this would fit into a scenario of disks nested within disks. Our data, with complete 2D coverage, favor best the nuclear outflow hypothesis, but in combination with streaming motions along nuclear spirals.

\subsection{The Origin of the Nuclear Spiral}

GB05 have used near-IR HST images of the nuclear region, where they found a stellar oval, in combination with the $\mathrm{CO}$ observations, in order to estimate whether torques produced by the nonaxisymmetric stellar potential would be efficient enough to drain the gas angular momentum, allowing it to flow into the nuclear region. GB05 conclude that the inflow is efficient only down to approximately the star-forming ring. Inside the ring, the torques are positive and cannot drive gas inflow. As molecular gas is observed much closer to the nucleus, GB05 conclude that inside the ring, other mechanisms should be responsible to drive the AGN fueling, such as viscous torques.

Englmaier \& Shlosman (2000) have used 2D numerical simulations to show that nuclear gaseous spirals are generated as a result of the gas response to the gravitational potential of a galactic disk with a large-scale stellar bar. Both NGC 6951 and NGC 1097 (Fathi et al. 2006) present similar large-scale stellar bars, which could thus play a role in the formation of the nuclear spirals.

More recently, Maciejewski (2004a, 2004b) has considered the effect of a SMBH in nuclear gaseous spirals. The fact that the SMBH does play a role is supported by the ubiquitous presence of nuclear spirals in Seyfert galaxies (Martini \& Pogge 1999; Pogge \& Martini 2002), and, in particular, by the strong correlation of their presence with the nuclear activity (Simões Lopes et al. 2007). Maciejewski concludes that if the asymmetry is strong, spiral shocks are produced, and the central SMBH can allow the spiral shocks to extend all the way to its immediate vicinity, generating a gas inflow of up to $0.03 M_{\odot} \mathrm{yr}^{-1}$. The cases of NGC 6951 presented here and NGC 1097 presented by Fathi et al. (2006) seem to support the models of Maciejewski (2004a, 2004b).

\subsection{Estimating the Mass Accretion Flow}

We can use the residual velocities observed along the nuclear spiral to estimate the rate of mass flowing to the nucleus. In order to do that, we assume that the mass flows along two nuclear spiral arms. The cross section of these arms can be estimated by assuming an opening angle of $20^{\circ}$ subtended by the spiral arms at $100 \mathrm{pc}$ from the nucleus. This opening angle corresponds to a radius for an assumed circular cross section of the spiral arm calculated as

$$
r \approx(100 \mathrm{pc}) \frac{10 \pi}{180}=17.5 \mathrm{pc}
$$

The flux of matter crossing the circular cross section can be calculated as

$$
\Phi=N_{e} v \pi r^{2} n_{\mathrm{arms}} m_{p} f
$$

where $N_{e}$ is the electron density, $v$ is the streaming velocity of the gas toward the nucleus, $n_{\text {arms }}$ is the number of spiral arms, $m_{p}$ is the proton mass, and $f$ is the filling factor. The filling factor was estimated from

$$
L_{\mathrm{H} \alpha} \sim f N_{e}^{2} j_{\mathrm{H} \alpha}(T) V
$$

where $L_{\mathrm{H} \alpha}$ is the $\mathrm{H} \alpha$ luminosity emitted by a volume $V$ and $j_{\mathrm{H} \alpha}(T)=3.534 \times 10^{-25} \mathrm{erg} \mathrm{cm}^{-3} \mathrm{~s}^{-1}$ (Osterbrock 1989). In the region covered by the partial spiral arms, we obtain an average flux per pixel (of angular size $0.1^{\prime \prime} \times 0.1^{\prime \prime}$ ) of $4.8 \times 10^{-17} \mathrm{erg} \mathrm{cm}^{-2}$ $\mathrm{s}^{-1}$. In order to calculate the $\mathrm{H} \alpha$ luminosity in the region covered by a partial arm, we adopt an area of $2^{\prime \prime} \times 1^{\prime \prime}$, which corresponds to 200 pixels. We thus obtain $L_{\mathrm{H} \alpha}=200\left(4.8 \times 10^{-17} \mathrm{erg} \mathrm{cm}^{-2}\right.$ $\left.\mathrm{s}^{-1}\right) 4 \pi(24 \mathrm{Mpc})^{2}=6.62 \times 10^{38} \mathrm{erg} \mathrm{s}^{-1}$. Adopting an approximate volume for the region (from Fig. 4) of $2^{\prime \prime} \times 1^{\prime \prime} \times 1^{\prime \prime}$, or $V=$ $2000(9.6 \mathrm{pc})^{3}$, we obtain $f \approx 0.004$.

The velocity residuals in the region of the partial nuclear arms, which we have interpreted as streaming motions, range from $\approx 20$ to $\approx 50 \mathrm{~km} \mathrm{~s}^{-1}$ (Fig. 4). Assuming that these streaming motions occur in the plane of the galaxy, we need to correct these values for the inclination of the galaxy, $i=42^{\circ}$. The resulting average velocity for the streaming motions is $\approx v=40 \mathrm{~km} \mathrm{~s}^{-1}$. For $N_{e}=$ $100 \mathrm{~cm}^{-3}$ (Fig. 3), $v=40 \mathrm{~km} \mathrm{~s}^{-1}$, and $n_{\text {arms }}=2$, we obtain a value for the flux of matter along the nuclear spirals of $\Phi \approx$ $0.004\left(1.23 \times 10^{25} \mathrm{~g} \mathrm{~s}^{-1}\right) \approx 8 \times 10^{-4} M_{\odot} \mathrm{yr}^{-1}$.

We note that the above calculation is only a rough estimate, as it depends on uncertain parameters, such as the geometry of the flow. Under the assumption that the gas moving along the spiral is reaching the nucleus, we can compare the above-calculated rate of ionized gas flow to the accretion rate necessary to reproduce the luminosity of the LINER nucleus of NGC 6951, which is calculated as follows. The nuclear luminosity can be estimated from the $\mathrm{H} \alpha$ luminosity of $L_{\mathrm{H} \alpha}=1.6 \times 10^{39} \mathrm{erg} \mathrm{s}^{-1}$ (from the nuclear flux and reddening quoted by P00), using the approximation that the bolometric luminosity is $L_{\mathrm{bol}} \approx 100 L_{\mathrm{H} \alpha}=1.6 \times$ $10^{41} \mathrm{erg} \mathrm{s}^{-1}$ (Ho 1999; Ho et al. 2001). This estimate is consistent also with the upper limit on the X-ray luminosity of this galaxy, $L_{X} \approx 4 \times 10^{40} \mathrm{erg} \mathrm{s}^{-1}$ (Fabbiano et al. 1992; Ho 1999). The mass accretion rate can then be estimated as

$$
\dot{m}=\frac{L_{\mathrm{bol}}}{c^{2} \eta}
$$

where $\eta$ is the efficiency of conversion of the rest mass energy of the accreted material into radiation. For a "standard" geometrically thin, optically thick accretion disk, $\eta \approx 0.1$ (e.g., Frank et al. 2002), but for LINERs it has been concluded that the accretion disk is geometrically thick and optically thin (Ho 2005; Nemmen et al. 2006; Yuan 2007). This kind of accretion flow is known as an RIAF (radiatively inefficient accretion flow; Narayan 2005) and has a typical value of $\eta \approx 0.01$. We use this value to derive an accretion rate of $\dot{m}=1.78 \times 10^{22} \mathrm{~g} \mathrm{~s}^{-1}$, or $2.8 \times 10^{-4} M_{\odot} \mathrm{yr}^{-1}$. Comparing this accretion rate, $\dot{m}$, with the mass flow of ionized gas along the nuclear spirals derived above, $\Phi$, we conclude that $\Phi \approx 3 \dot{m}$. Thus, the flow of ionized gas along the spiral arms is on the order of the one necessary to feed the AGN in NGC 6951.

We should nevertheless point out that this flow is most probably a lower limit to the actual inward flow of matter, as we are observing only the ionized gas, which may be just the tip of the iceberg. Neutral and molecular gas may also be flowing in, as suggested by the $\geq 10^{7} M_{\odot}$ of molecular gas mass detected at the nucleus by GB05 and Krips et al. (2007).

We now compare the above results obtained for NGC 6951 with those obtained for NGC 1097. Storchi-Bergmann (2007) has calculated the mass flow rate along the nuclear spirals of NGC 1097 on the basis of similar IFU observations, but for a filling factor of $f=1$. If we adopt a more realistic value of $\sim 10^{-3}$, it can be concluded that the mass flow rate of ionized gas is again similar to the accretion rate necessary to power the LINER nucleus of 
NGC 1097. Similarly to the case of NGC 6951, the total mass flow is probably larger and could include neutral and molecular gas. A larger mass flow rate would be consistent with the presence of a young starburst (age $\sim 10^{6} \mathrm{yr}$ ) with mass $10^{6} M_{\odot}$ that was discovered in the nuclear HST STIS UV nuclear spectrum of NGC 1097 and is estimated to be closer than $9 \mathrm{pc}$ from the nucleus (Storchi-Bergmann et al. 2005). The gas flowing to the nuclear region is thus not only feeding the nucleus, but has also accumulated enough mass to give origin to a circumnuclear starburst in the last $10^{6} \mathrm{yr}$. The association of recent star formation with nuclear activity has been claimed in many recent studies (e.g., Cid Fernandes et al. 2001; Storchi-Bergmann et al. 2001), and its association in particular with dust structures in early-type galaxies has been also supported by the recent work of Ferrarese et al. (2006).

\section{CONCLUDING REMARKS}

We have presented IFU-GMOS observations of the ionized gas in the inner region of the galaxy NGC 6951 that evidence the presence of streaming motions along nuclear spiral arms toward its LINER nucleus. The streaming motions are observed over an extent of $\approx 200 \mathrm{pc}$ along two partial spiral arms down to $\approx 100 \mathrm{pc}$ from the nucleus.

This is so far only the second galaxy in which such streaming motions have been mapped, the first being NGC 1097, which was also mapped by our group (Fathi et al. 2006). The relevance of this result stems from the fact that the presence of dusty nuclear structures correlates strongly with nuclear activity (Simões Lopes et al. 2007), which suggests that these structures are associated with the actual fuel on its way in to feed the nuclear SMBH.

Nevertheless, until recently, there were no kinematic measurements that showed that these spirals do carry gas inward. Such kinematic measurements are difficult to obtain due to the fact that the gas kinematics of the nuclear region of active galaxies is usually dominated by outflows from the narrow-line region. Most kinematic studies do reveal outflows and no inflows (e.g., Crenshaw \& Kraemer 2007 and references therein).

In order to observe colder gas that can fall inward, it is necessary to obtain gas kinematics around nuclei with low levels of activity, such that the outflows are small, if present. This seems to be the case of NGC 6951: a LINER nucleus, with a small jet, such that it was possible to disentangle the outflow related to the jet from streaming motions along nuclear spirals. Additional observational constraints to observe such motions around a galaxy nucleus include a favorable orientation of the galaxy to allow the measurement of the kinematics and strong enough gas emission to allow such measurements up to several hundred parsecs from the nucleus, so that we can constrain the disk rotation and on top of it measure the residuals from circular motion.

With the present results for NGC 6951, we can now say that there are two galaxies with confirmed inward streaming motions along nuclear spirals, NGC 1097 and NGC 6951, giving strong support to the hypothesis that these spirals are indeed channels through which the matter flows inward to feed the SMBH.

The kinematic measurements and an estimated geometry derived from the observations has allowed us to estimate the ionized gas mass flow rate along the nuclear spirals of NGC 1097 and NGC 6951. We obtain values that are similar to the accretion rate necessary to power the AGN. The total mass flow rate is most probably larger, as it would include neutral and molecular gas. In the case of NGC 6951, molecular gas has been recently observed both at the star-forming ring and inside the ring. In the case of NGC 1097, the young nuclear starburst found by Storchi-Bergmann et al. (2005) also supports a larger flow of matter to the nucleus in the last $10^{6} \mathrm{yr}$. Thus, it may be that the inflows observed along nuclear spiral arms not only feed nuclear SMBHs, but at the same time contribute to the growth of the bulge via star formation. As a result, both the stellar component of the galaxy and its SMBH at the nucleus grow after each activity cycle, in agreement with the scenario implied by the $M_{\mathrm{BH}}-\sigma$ relation (e.g., Tremaine et al. 2002).

We acknowledge the referee for relevant suggestions that have improved the paper. This work was based on observations obtained at the Gemini Observatory, which is operated by the Association of Universities for Research in Astronomy, Inc., under a cooperative agreement with the NSF on behalf of the Gemini partnership: the National Science Foundation (USA), the Particle Physics and Astronomy Research Council (UK), the National Research Council (Canada), CONICYT (Chile), the Australian Research Council (Australia), CNPq (Brazil), and CONICET (Argentina). K. F. acknowledges support from the Werner-Gren Foundations, the Royal Swedish Academy of Sciences, and project P3/86 from the Instituto de Astrofísica de Canarias. This work is also based on observations with the NASA/ESA Hubble Space Telescope obtained at the Space Telescope Science Institute, which is operated by the Association of Universities for Research in Astronomy, Inc., under NASA contract NAS5-26555. T. S.-B., O. L. D., and R. A. R. acknowledge support from the Brazilian institutions CNPq and CAPES.
Allington-Smith, J., et al. 2002, PASP, 114, 892

Binney, J., \& Merrifield, M. 1998, Galactic Astronomy (Princeton: Princeton Univ. Press)

Binney, J., \& Tremaine, S. 1987, Galactic Dynamics (Princeton: Princeton Univ. Press)

Cid Fernandes, R., Heckman, T., Schmitt, H., González Delgado, R. M., \& Storchi-Bergmann, T. 2001, ApJ, 558, 81

Crenshaw, D. M., \& Kraemer, S. B. 2007, ApJ, 659, 250

Crenshaw, D. M., Kraemer, S. B., \& Gabel, J. R. 2003, AJ, 126, 1690

Emsellem, E., Fathi, K., Wozniak, H., Ferruit, P., Mundell, C., \& Shinnerer, E. 2006, MNRAS, 365, 367

Emsellem, E., Goudfrooij, P., \& Ferruit, P. 2003, MNRAS, 345, 1297

Emsellem, E., et al. 2001, A\&A, 368, 52

Englmaier, P., \& Shlosman, I. 2000, ApJ, 528, 677 2004, ApJ, 617, L115

Erwin, P., \& Sparke, L. S. 1999, in ASP Conf. Ser. 182, Galaxy Dynamics, ed. D. R. Merritt, M. Valluri, \& J. A. Sellwood (San Francisco: ASP), 243

Fabbiano, G., Kim, D.-W., \& Trinchieri, G. 1992, ApJS, 80, 531

Fathi, K., Storchi-Bergmann, T., Riffel, R. A., Winge, C., Axon, D. J., Robinson, A., Capetti, A., \& Marconi, A. 2006, ApJ, 641, L25

\section{REFERENCES}

Fathi, K., van de Ven, G., Peletier, R. F., Emsellem, E., Falcón-Barroso, J., Cappellari, M., \& de Zeeuw, T. 2005, MNRAS, 364, 773

Ferrarese, L., et al. 2006, ApJS, 164, 334

Filippenko, A. V., \& Sargent, W. L. W. 1985, ApJS, 57, 503

Frank, J., King, A. R., \& Raine, D. J. 2002, Accretion Power in Astrophysics

(3rd ed.; Cambridge: Cambridge Univ. Press)

Freeman, K. 1970, ApJ, 160, 811

Garcia-Burillo, S., Combes, F., Schinnerer, E., Boone, F., \& Hunt, L. K. 2005, A\&A, 441, 1011 (GB05)

Ho, L. C. 1999, ApJ, 516, 672

. 2005, Ap\&SS, 300, 219

Ho, L. C., Filippenko, A. V., \& Sargent, W. L. W. 1997, ApJS, 112, 315

Ho, L. C., \& Ulvestad, J. S. 2001, ApJS, 133, 77

Ho, L. C., et al. 2001, ApJ, 549, L51

Keppel, J. W., et al. 1991, ApJ, 374, 507

Knapen, J. H. 2005, Ap\&SS, 295, 85

Knapen, J. H., et al. 2000, ApJ, 528, 219

Kohno, K., Kawabe, R., Shibatsuka, T., \& Matsushita, S. 2000, in ASP Conf. Proc. 217, Imaging at Radio through Submillimeter Wavelengths, ed. J. G. Mangum \& S. J. E. Radford (San Francisco: ASP), 364 
Krips, M., et al. 2007, A\&A, 468, L63

Laine, S., et al. 2003, AJ, 126, 2717

Maciejewski, W. 2004a, MNRAS, 354, 883 2004b, MNRAS, 354, 892

Maciejewski, W., Teuben, P. J., Sparke, L. S., \& Stone, J. M. 2002, MNRAS, 329,502

Marconi, A., et al. 2003, ApJ, 586, 868

Márquez, I., \& Moles, M. 1993, AJ, 105, 2090

Martini, P., \& Pogge, R. W. 1999, AJ, 118, 2646

Martini, P., Regan, M. W., Mulchaey, J. S., \& Pogge, R. W. 2003, ApJ, 589, 774

Narayan, R. 2005, in From X-ray Binaries to Quasars: Black Holes on All Mass Scales, ed. T. J. Maccarone, R. P. Fender, \& L. C. Ho (Dordrecht: Kluwer), 177

Nemmen, R. S., Storchi-Bergmann, T., Yuan, F., Terashima, Y., \& Wilson, A. S. 2006, ApJ, 643, 652

Osterbrock, D. E. 1989, Astrophysics of Gaseous Nebulae and Active Galactic Nuclei (Mill Valley: University Science Books)

Pérez, E., Márquez, I., Marrero, I., Durret, F., González Delgado, R. M., Masegosa, J., Maza, J., \& Moles, M. 2000, A\&A, 353, 893 (P00)

Pogge, R. W., \& Martini, P. 2002, ApJ, 569, 624

Riffel, R., Storchi-Bergmann, T., Winge, C., \& Barbosa, F. K. B. 2006, MNRAS, 373,2
Saikia, D. J., Ohookun, B., Pedlar, A., \& Kohno, K. 2002, A\&A, 383, 98

Saikia, D. J., Pedlar, A., Unger, S. W., \& Axon, D. J. 1994, MNRAS, 270, 46

Shlosman, I., Begelman, M. C., \& Frank, J. 1990, Nature, 345, 679

Simões Lopes, R. D., Storchi-Bergmann, T., Martini, P., \& Saraiva, M. F. 2007, ApJ, 655, 718

Sokolowski, J., Bland-Hawthorn, J., \& Cecil, G. 1991, ApJ, 375, 583

Storchi-Bergmann, T. 2007, in Proc. IAU Symp. 238, Black Holes from Stars to Galaxies - Across the Range of Masses, ed. V. Karas \& G. Matt (Cambridge: Cambridge Univ. Press), 283

Storchi-Bergmann, T., González Delgado, R. M., Schmitt, H. R., Cid Fernandes, R., \& Heckman, T. 2001, ApJ, 559, 147

Storchi-Bergmann, T., Nemmen, R. S., Spinelli, P. F., Eracleous, M., Wilson, A. S., Filippenko, A. V., \& Livio, M. 2005, ApJ, 624, L13

Storchi-Bergmann, T., Wilson, A. S, \& Baldwin, J. A. 1996, ApJ, 460, 252

Sutherland, R. S., Bicknell, G. V., \& Dopita, M. 1993, ApJ, 414, 510

Tremaine, S., et al. 2002, ApJ, 574, 740

Tully, R. B. 1988, Nearby Galaxies Catalog (Cambridge: Cambridge Univ. Press) Viegas, S. M., \& Contini, M. 1994, ApJ, 428, 113

Yuan, F. 2007, in ASP Conf. Ser. 373, The Central Engine of Active Galactic Nuclei, ed. L. C. Ho \& J.-M. Wang (San Francisco: ASP), 95 